\title{
Conditioned pain modulation is minimally influenced by cognitive evaluation or imagery of the conditioning stimulus
}

This article was published in the following Dove Press journal:

Journal of Pain Research

26 November 2014

Number of times this article has been viewed

\section{Mario Bernaba \\ Kevin A Johnson \\ Jiang-Ti Kong \\ Sean Mackey}

Stanford Systems Neuroscience and Pain Laboratory, Department of Anesthesiology, Perioperative and Pain Medicine, Stanford University School of Medicine, Stanford, CA, USA
Correspondence: Sean Mackey Department of Anesthesiology, Perioperative and Pain Medicine, Division of Pain Management, Stanford University Medical School 1070 Arastradero Rd, Suite 200, Palo Alto, CA 94304, USA

$\mathrm{Tel}+\mathrm{I} 6504986477$

Fax +l 6507259642

Email smackey@stanford.edu
Purpose: Conditioned pain modulation (CPM) is an experimental approach for probing endogenous analgesia by which one painful stimulus (the conditioning stimulus) may inhibit the perceived pain of a subsequent stimulus (the test stimulus). Animal studies suggest that CPM is mediated by a spino-bulbo-spinal loop using objective measures such as neuronal firing. In humans, pain ratings are often used as the end point. Because pain self-reports are subject to cognitive influences, we tested whether cognitive factors would impact on CPM results in healthy humans.

Methods: We conducted a within-subject, crossover study of healthy adults to determine the extent to which CPM is affected by 1) threatening and reassuring evaluation and 2) imagery alone of a cold conditioning stimulus. We used a heat stimulus individualized to $5 / 10$ on a visual analog scale as the testing stimulus and computed the magnitude of CPM by subtracting the postconditioning rating from the baseline pain rating of the heat stimulus.

Results: We found that although evaluation can increase the pain rating of the conditioning stimulus, it did not significantly alter the magnitude of CPM. We also found that imagery of cold pain alone did not result in statistically significant CPM effect.

Conclusion: Our results suggest that CPM is primarily dependent on sensory input, and that the cortical processes of evaluation and imagery have little impact on CPM. These findings lend support for CPM as a useful tool for probing endogenous analgesia through subcortical mechanisms.

Keywords: conditioned pain modulation, endogenous analgesia, evaluation, imagery, cold presser test, CHEPS, contact heat-evoked potential stimulator

\section{Introduction}

Conditioned pain modulation (CPM) is an experimental tool for measuring endogenous analgesia in humans. ${ }^{1}$ It is often linked to mechanisms first explored in animal research. In rats, Le Bars et $\mathrm{al}^{2}$ demonstrated that one noxious stimulus (the conditioning stimulus) could inhibit the neural response to another noxious stimulus (the test stimulus) from a distal location. These animal studies implicate a spino-bulbo-spinal loop in the process, whereby wide dynamic range neurons in the spinal dorsal horn receive a noxious conditioning stimulus from one body part and send a signal upward to the subnucleus reticularis dorsalis of the caudal medulla, which then sends widespread descending inhibition to spinal secondary neurons via the dorsolateral funiculi. ${ }^{3,4}$ Researchers have used various terms to describe this phenomenon, including diffuse noxious inhibitory controls, counterirritation, and heterotopic noxious conditioning stimulation. ${ }^{5}$ To standardize terminology, experts recommended strictly using diffuse 
noxious inhibitory controls to describe the lower brainstemmediated inhibitory mechanism directly observed in animal studies, and using CPM to describe the human behavioral correlate. $^{6}$

A standard CPM paradigm consists of a single conditioning stimulus and a test stimulus that is measured twice, once before and once during or after the application of the conditioning stimulus. CPM is typically characterized by the change in the pain rating of the testing stimulus as a result of the application of the conditioning stimulus. ${ }^{5}$ Assessing CPM in humans facilitates the understanding of the underlying mechanisms of chronic pain conditions ${ }^{7-9}$ and helps predict their response to treatment. ${ }^{1,10,11}$

Because CPM relies on self-reports of pain perception, we sought to understand how higher cortical mechanisms that influence self-report may impact CPM. We specifically focused our study on the conditioning stimulus, the intensity of which has been shown to affect the magnitude of CPM. ${ }^{12-14}$ We used a CPM protocol where the conditioning stimulus was a cold water bath ${ }^{15-18}$ and the testing stimulus was noxious contact heat, both adjusted to the individual's level of moderate pain.

Our first aim was to determine whether cognitive evaluation of the conditioning stimulus would impact 1 ) the perceived pain of the conditioning stimulus and 2) the magnitude of CPM. We identified a prior study that manipulated evaluation (threatening and reassuring) to impact pain tolerance of a cold bath, ${ }^{19}$ and we assessed whether such an evaluation on a cold conditioning stimulus could impact the heat test stimulus rating in CPM. In the prior study, the manipulated evaluation changed the tolerance of the cold water bath but did not significantly change the pain ratings of the cold water bath.

Our second aim was to test whether using imagery of cold pain alone as the conditioning stimulus (no sensory stimulus) would reduce the pain rating of the testing stimulus. Nociception is not always necessary or sufficient for the perception of pain, and some studies have reported that noxious stimuli are not required for CPM. ${ }^{8,12}$ We thus further investigated whether mental imagery alone, in lieu of physical stimuli, is able to induce a CPM-like effect. Assuming that higher cortical mechanisms play a role in CPM, we hypothesized that imagery alone could generate a CPM-like response.

\section{Methods}

\section{Participants}

The Stanford University Institutional Review Board reviewed and approved all procedures. We recruited healthy participants between the ages of 18 years and 45 years from the local community through internet advertisements. We excluded volunteers for medication use, the presence of a pain condition, any circulatory disorder, a previous cold injury, problems with blood clotting, or any skin condition. A total of 20 participants were recruited, with an equal number of males and females. They provided informed consent prior to the start of experimental procedures.

\section{Experimental design - task flow}

Figure 1 summarizes the tasks performed by our participants in temporal order. First, they completed a heat pain thresholding procedure to individualize the temperature for the CPM heat test stimulus. At the end of the thresholding procedure, participants provided a baseline pain rating of their individualized heat stimulus on their left hand. This rating would be used in all subsequent CPM calculations as the baseline "before rating" of the test stimulus. Next, they performed the first imagery task (precold water experience), imagining their right foot in cold water for 2 minutes while the heat test stimulus was applied to their left hand during the last 30 seconds of the imagery. Next, they completed a cold pain thresholding procedure in an actual cold water bath, to individualize the temperature for the CPM conditioning stimulus. Subsequently, participants completed a baseline CPM procedure using their individualized cold water conditioning stimulus and individualized heat test stimulus. Finally, in randomized order, each participant completed two more CPM procedures involving either a threatening or a reassuring evaluation of the cold water conditioning stimulus and a second imagery task (postcold water experience).

\section{Individual thresholding of the heat test stimulus}

We administered heat using a Pathway system (Medoc Advanced Medical Systems, Ramat Yishai, Israel), with a contact heat-evoked potential stimulator thermode secured on the left palm. Participants rated their pain using a computerized visual analog scale (VAS), with a left anchor of "no pain" (recorded as 0/10) and a right anchor of "worst pain imaginable" (recorded as 10/10).

We found the temperature that gave approximately a 5/10 pain rating for each participant ("Heat-5") and applied this temperature on the palm for 30 seconds as our test stimulus. To identify Heat-5, we first obtained a thermal maximum by collecting pain ratings from $32.0^{\circ} \mathrm{C}$ to $51.0^{\circ} \mathrm{C}$, increasing at a rate of $0.3^{\circ} \mathrm{C} /$ second. The participant indicated if the temperature reached "worst pain imaginable", and we set the 


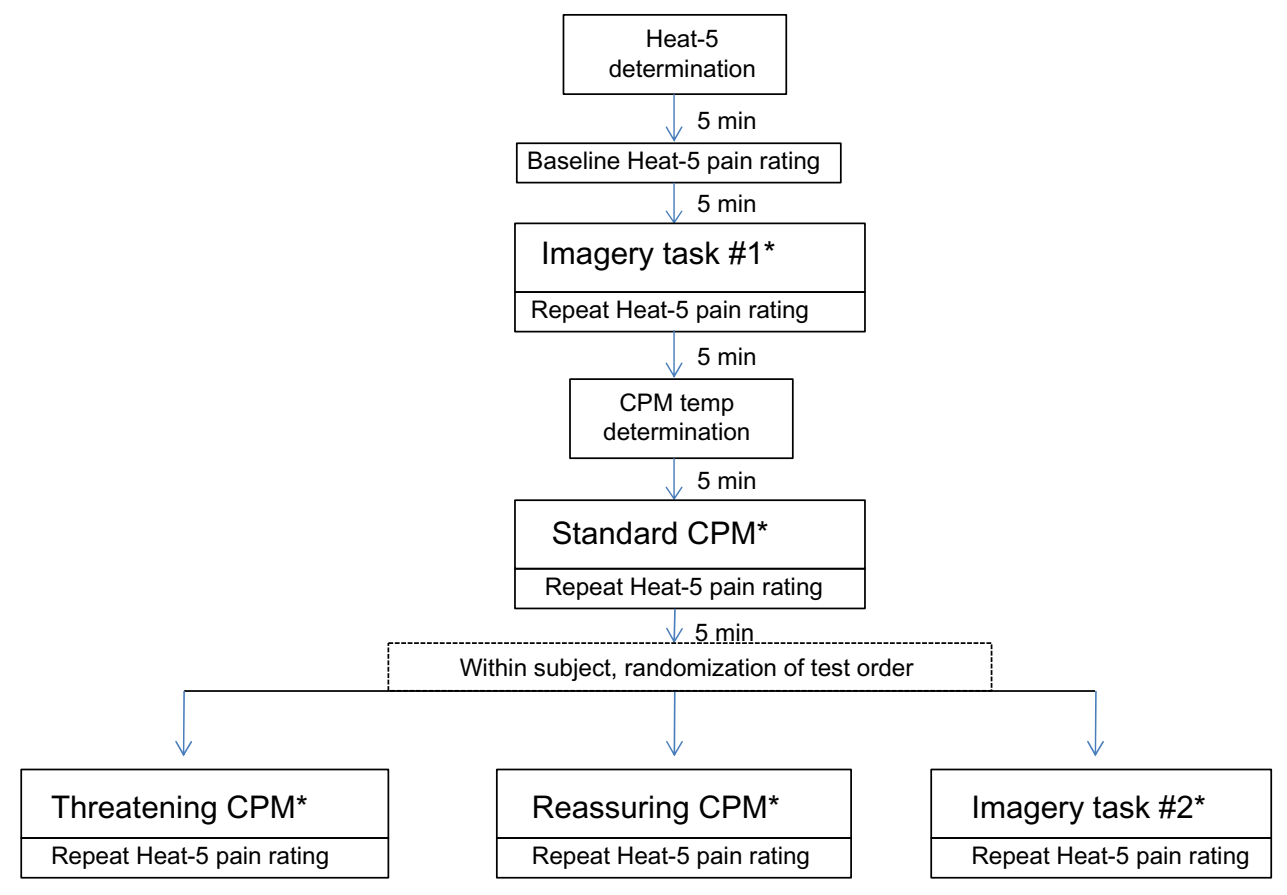

Figure I Experimental outline - task flow.

Notes: All tasks with “*”are 120 seconds long and were applied to the right foot. During the last 30 seconds of the 120 seconds, the Heat-50 (test stimulus) is applied to the left hand. The rating of the repeat Heat-5 occurs at the end of the 30 seconds. Task \#I refers to pre-cold water exposure imagery, and task \#2 refers to post-cold water exposure imagery.

Abbreviation: CPM, conditioned pain modulation; min, minutes.

thermal maximum at the maximally tolerated temperature averaged from two runs repeated 1 minute apart. Next, a method-of-limit test ${ }^{20}$ was used to find Heat-5. Specifically, the participant was subjected to a series of 13 temperatures for 30 seconds each, with 30 -second breaks, to rate the pain of the thermode stimulus using the VAS. The 13 temperatures ranged from $1{ }^{\circ} \mathrm{C}$ and $5.5^{\circ} \mathrm{C}$ below the thermal maximum, with $48.9^{\circ} \mathrm{C}$ being the maximum temperature allowed for 30 seconds, for safety considerations. We used the participant's 13 pain ratings to estimate the test stimulus that would yield a $5 / 10$ pain rating.

Finally, we performed a baseline heat test where Heat-5 was applied to the participant's palm for 30 seconds. We repeated the heat pain thresholding if the baseline rating of the current Heat-5 resulted in pain ratings beyond a VAS range of 4-7. We excluded participants if we were unable to find a stable temperature setting that yielded a baseline heat test rating within the range of 4/10-7/10.

\section{Imagery of cold pain protocol}

Participants performed cold imagery both before and after actual exposure. Before the application of any real cold conditioning stimulus, we tested whether the mental imagery of cold pain alone would impact the heat test pain rating. The participants listened to a recorded script that guided them to imagine their right foot in painfully cold water for 120 seconds (Script 1 in Supplementary materials). We applied the 30 -second heat test stimulus starting at 90 seconds into the mental imagery tasks, and the participants rated the heat test stimulus on the VAS immediately following the task. They also rated how well they thought they could perform the imagery task on a VAS scale ( $0=$ not all, $10=$ like it was real $)$. We then repeated this imagery task again after exposure to the actual cold bath using both the initial cold bath thresholding and a standard CPM protocol (details in Standard CPM protocol section). We specifically performed this imagery twice to assess whether exposure to the actual cold conditioning stimulus would influence the quality of the imagery task and, subsequently, the magnitude of CPM.

\section{Individual thresholding of the cold water conditioning stimulus}

To set the cold water temperature for the CPM conditioning stimulus, we used a rough step-down thresholding procedure to find the temperature that approximated a $5 / 10$ pain rating. Five minutes after the first imagery of the cold pain task, the participants placed their right foot into a circulating cold 
water container set to three temperatures $\left(16^{\circ} \mathrm{C}, 12^{\circ} \mathrm{C}\right.$, and $8^{\circ} \mathrm{C}$ ) for 120 seconds each, with 2 minutes between each exposure. The participants rated their pain at 60 seconds and 120 seconds on the VAS, and we averaged the results. We selected the temperature for the cold water bath that resulted in a mean pain rating closest to $5 / 10$.

\section{Standard CPM protocol}

The study participants completed a standard CPM protocol approximately 5 minutes after the thresholding of the cold water conditioning stimulus. The cold water conditioning stimulus lasted for 120 seconds as participants placed their right foot in the circulating cold water container with a temperature determined by individualized thresholding as described in the preceding section. The 30 -second heat was applied to the left palm between 90 seconds and 120 seconds of cold water exposure. After being exposed to both stimuli for 30 seconds, the participants removed their foot from the bath and separately rated the pain produced by the conditioning stimulus and pain of the test stimulus.

\section{Threatening and reassurance evaluation protocol}

Approximately 5 minutes following the standard CPM trial, the participants performed two modified CPM trials involving evaluation of the cold water conditioning stimulus. Prior to these modified CPM runs, they listened to a recorded script guiding them to evaluate the cold water conditioning stimulus as threatening in one trial and as safe in the other trial (adapted from Jackson et al: ${ }^{19}$ Script 2 and 3 in Supplementary materials). Then the standard CPM protocol was run, modified with participants instructed to perform either the threatening or the reassurance evaluation of the conditioning stimulus. The threatening and reassurance evaluation trials were randomized with the second imagery trial (postcold water exposure). Each trial was separated by 5 minutes.

\section{Data analysis}

We completed data analysis using SPSS version 20 (IBM, Armonk, NY, USA). We used linear mixed-effects modeling for primary comparisons ${ }^{21}$ because this approach was capable of accounting for the characteristics of our data set, including the handling of within-individual repeated measures. We also used paired-samples $t$-tests and the bivariate correlation analysis for secondary exploration of our data. $\mathrm{G}^{*}$ Power version 3.1.3 was used to compute the sample size needed based on our results, to detect significant differences in pain ratings of the heat stimulus as a result of imagery.

\section{Results}

\section{Demographics and individualized thermal}

\section{settings}

We collected data from 20 healthy volunteers (ten women, ten men). Ages ranged from 18 years to 42 years (mean 25.1 years). We included 15 of those volunteers in the data analysis (eight women, seven men; mean 24.6 years). We excluded five participants because of issues related to the thresholding of the heat test stimulus. Four individuals did not achieve at least a $4 / 10$ pain rating on the maximum baseline heat test stimulus $\left(48.9^{\circ} \mathrm{C}\right)$. Low-level pain ratings would introduce floor effects, and safety considerations prevented us from applying higher temperatures to increase the pain rating. The fifth excluded participant rated the baseline heat test higher than $7 / 10$. This rating indicated that the thresholding was unstable, with likely hypersensitivity due to repeated testing alone. Table 1 lists the individualized temperature settings of the heat stimulus and cold water bath for each participant.

\section{Effect of cold water evaluation on the conditioning cold water pain rating}

To assess the effect of cold water evaluation on the participant's VAS rating of the cold water pain, we ran a linear

Table I Individualized temperature settings for the test and conditioning stimuli ${ }^{\mathrm{a}}$

\begin{tabular}{lll}
\hline $\begin{array}{l}\text { Subject } \\
\text { ID }\end{array}$ & $\begin{array}{l}\text { Heat-5 } \\
\left({ }^{\circ} \mathbf{C}\right)\end{array}$ & $\begin{array}{l}\text { Cold bath } \\
\text { temperature }\left({ }^{\circ} \mathbf{C}\right)\end{array}$ \\
\hline 02 & 46.5 & 16 \\
03 & 48 & 8 \\
04 & 44 & 16 \\
05 & 48.9 & 8 \\
06 & 48.9 & 16 \\
07 & 46 & 8 \\
08 & 45.4 & 8 \\
09 & 45.5 & 12 \\
11 & 45.6 & 8 \\
12 & 47.5 & 8 \\
14 & 47.3 & 8 \\
15 & 43.5 & 12 \\
16 & 46.5 & 8 \\
17 & 46.3 & 8 \\
19 & 44.5 & 16 \\
\hline
\end{tabular}

Notes: aWe used Heat- 5 as the test stimulus, applied via a Medoc contact heatevoked potential stimulator thermode to the left palm during the last 30 seconds of the application of conditioning stimulus. We used a cold water bath as the conditioning stimulus, applied to the entire right foot for 2 minutes. 
mixed-effects model for the random effect of the cold water pain rating by the fixed effect of the cold water evaluation (no evaluation with standard CPM, threatening evaluation with modified CPM, and reassurance evaluation with modified CPM). An autoregressive covariance matrix was added for within-subject repeated measures.

As shown in Figure 2, the modeled mean rating of cold water pain was 4.16 (standard error $[\mathrm{SE}]=0.50$ ) with no evaluation, 4.55 ( $\mathrm{SE}=0.78$ ) with threatening evaluation, and $3.46(\mathrm{SE}=0.42)$ with reassurance evaluation. Evaluation had a significant effect, $F(2,26.7)=3.37, P=0.05$, on the pain rating of the cold water conditioning stimulus. Furthermore, we did not find a modeled significant difference between the baseline and threatening conditions, $t(27.6)=-0.77, P=0.45$, but reassurance was significantly less than threatening, $t(25.2)=-2.58, P=0.02$. The participants rated their ability to perform the reassurance evaluation with a mean of 6.56 (standard deviation $[\mathrm{SD}]=2.43$ ) and the threatening evaluation with a mean of $6.04(\mathrm{SD}=1.49)$. We did not find a significant difference of these means by paired-samples $t$-test, $t(14)=0.82, P=0.42$.

\section{Effect of cold water evaluation on the heat test stimulus rating}

To assess the effect of the cold water evaluation on the postconditioning rating of the heat test stimulus pain, we ran a linear mixed-effects model to determine the random effect of the heat test pain rating by the fixed effect of the cold water evaluation (no evaluation with standard CPM, threatening evaluation with modified CPM, and reassurance evaluation with modified CPM). An autoregressive covariance matrix was added for within-subject repeated measures.

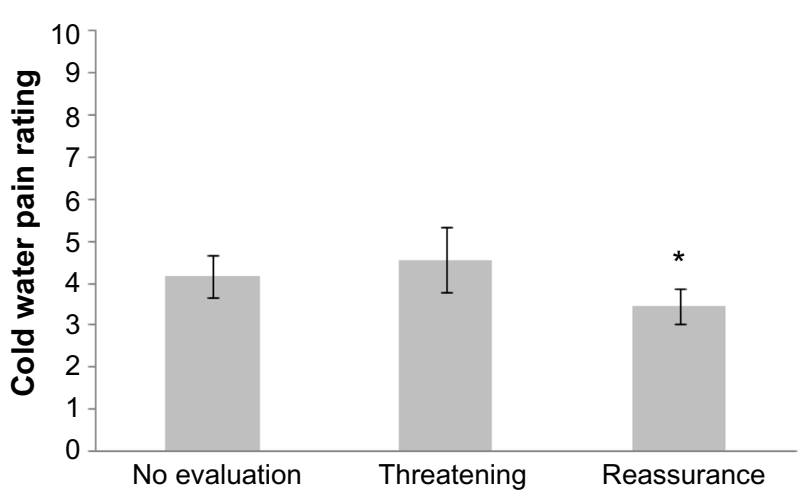

Figure 2 Cold water pain ratings for evaluation of the cold water. ${ }^{a}$

Notes: ${ }^{\mathrm{W}} \mathrm{We}$ failed to find a significant difference in the rating of the conditioning stimulus between baseline and threatening or reassuring conditions, $t(27.6)=-0.77, P=0.45$. However, reassurance was significantly less than threatening, $t(25.2)=-2.58, P=0.02$. *The reassuring condition was significantly different from the threatening condition but neither was significantly different from no evaluation.

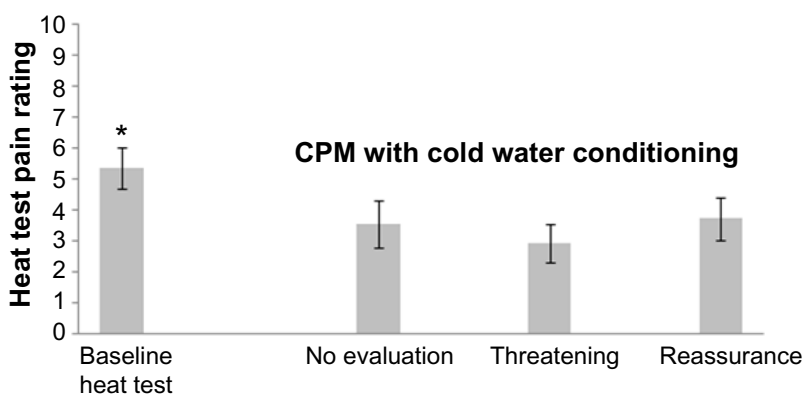

Figure 3 Heat test pain ratings for evaluation of the cold water.a

Notes: ${ }^{a}$ We failed to find any difference in the post-conditioning rating of the test stimulus among no evaluation, threatening, or reassurance. Hence, evaluation did not affect the magnitude of conditioned pain modulation (CPM), which is calculated by subtracting the post-conditioning rating from the baseline rating of the test stimulus, $F(2,28.5)=0.70, P=0.50$. *The difference was statistically significant between the baseline pain rating and any of the post-conditioning pain ratings of the test stimulus, regardless of the presence and mode of evaluation.

As shown in Figure 3, the modeled mean rating was 3.52 $(\mathrm{SE}=0.76)$ for the heat test pain with no evaluation, 2.92 $(\mathrm{SE}=0.64)$ for the threatening evaluation, and $3.72(\mathrm{SE}=0.70)$ for the reassurance evaluation. The cold water evaluation did not significantly affect the postconditioning rating of the heat test stimulus, $F(2,28.5)=0.70, P=0.50$.

\section{Can cold pain imagery result in CPM?}

To assess whether imagery can lead to a CPM-like effect, we ran a linear mixed-effects model to determine the random effect of postconditioning heat pain rating by the fixed effect of conditioning stimulus (no conditioning stimulus at baseline heat test, cold water conditioning stimulus across CPM trials, and cold pain imagery conditioning), adding an autoregressive covariance matrix for within-subject repeated measures.

As shown in Figure 4, the modeled mean rating of heat pain was $5.35(\mathrm{SE}=0.66)$, at the baseline test was $3.46(\mathrm{SE}=0.48)$

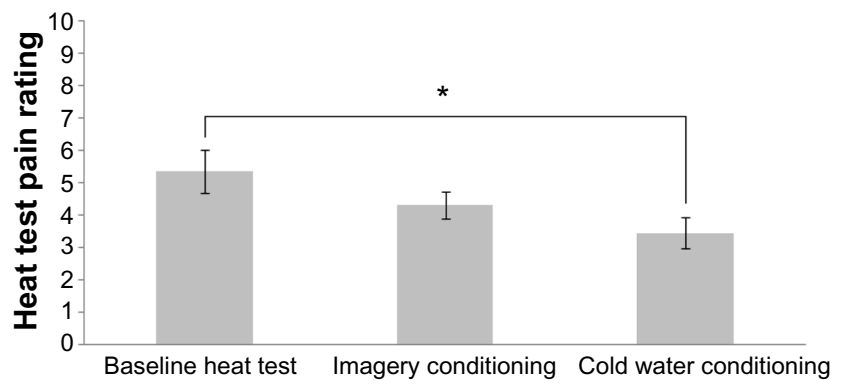

Figure 4 Pain ratings for baseline heat test, heat test with imagery conditioning, and heat test with cold water conditioning. ${ }^{\text {a }}$

Notes: ${ }^{a}$ We did not find a significant effect of imagery conditioning on the pain rating of the test stimulus. In contrast, cold water conditioning resulted in significant reduction in the pain rating of the test stimulus, $t(86.4)=-2.76, P=0.01$. However, there was no significant difference in heat test pain ratings for the cold pain imagery trials as compared with baseline, $t(75.8)=1.54, P=0.13$, or from CPM trials, $t(65.3)=-$ I.8I, $P>0.08$. *Cold water conditioning resulted in a significant reduction in the pain rating of the pain stimulus, whereas the pain rating from imagery conditioning was not significantly different from either the baseline or the cold water conditioning. 
across CPM trials, and was 4.33 (SE =0.42) across cold pain imagery trials. The conditioning stimulus had a significant effect, $F(2,73.6)=4.09, P=0.02$. The heat test pain ratings were significantly lower for CPM trials relative to the baseline heat test, $t(86.4)=-2.76, P=0.01$. However, the heat test pain ratings after cold pain imagery were not statistically different from baseline, $t(75.8)=1.54, P=0.13$, or from the cold water trials, $t(65.3)=-1.81, P=0.08$.

Next, we compared the first (precold water experience) to the second (postcold water experience) imagery task. Participants rated their ability to perform the first imagery task with a mean of $5.35(\mathrm{SD}=1.89)$ and the second with a mean of $6.47(\mathrm{SD}=1.82)$. We failed to find a significant difference in these means using a paired-samples $t$-test, $t(14)=2.04, P=0.06$. The mean postimagery heat pain rating was $4.22(\mathrm{SD}=2.4)$ for the first trial and $4.52(\mathrm{SD}=2.7)$ for the second trial. They were not significantly different, $t(14)=0.37, P=0.72$.

\section{Discussion}

In this study, we replicated CPM in a healthy cohort using a cold water bath as the conditioning stimulus and contact heat pain as the test stimulus. We also replicated the finding that cognitive evaluation of a cold pain stimulus can alter the experience of that stimulus. ${ }^{19}$ More importantly, we found that 1) cognitive evaluation of the conditioning stimulus did not significantly change the magnitude of CPM, despite altering the pain rating of the conditioning stimulus itself, and 2) using cold pain imagery in lieu of an actual cold pain stimulus did not result in a statistically significant CPM-like effect. These results suggest that CPM is more dependent on physical conditioning stimuli than on higher cognitive and evaluative processes. Here, we interpret our results in detail and compare them with existing literature.

First, we discovered that cognitive evaluation of a fixed cold stimulus can alter the rating of the resulting cold pain (Figure 2). Our finding is consistent with prior findings in the literature that indicated that a number of cognitive factors have been shown to alter the perception of pain. Examples of such factors include appraisal, fear, and distraction. ${ }^{19,22-24}$ Moreover, we did not find a statistically significant difference in the rating of the cold stimulus between no evaluation and threatening evaluation (Figure 2). This result suggests that cold water may be naturally perceived as threatening for our population sample, and this threat can be reduced through reassurance.

Second, although evaluation influenced the pain perception of the conditioning stimulus, it did not lead to statistically significant changes in the magnitude of CPM in our sample (Figure 3). This finding highlights the robustness of CPM compared with a simple measure of the cold pain, which is subject to evaluative influences. Our result is also consistent with prior literature findings that indicate that the subjective pain ratings of the conditioning stimulus do not predict CPM magnitude. . $^{5,12,14,16,25}$

Third, we found that imagery, in lieu of a physical conditioning stimulus, did not cause a statistically significant reduction in the pain ratings of the test stimulus (and therefore did not result in a significant CPM effect). This result is consistent with the findings of a majority of the studies that suggest that a noxious physical stimulus is required to obtain CPM. ${ }^{16,26-28}$ The few studies that indicated that noxious stimuli were not required ${ }^{8,12}$ used a long conditioning stimulus ( $>5$ minutes), which may have potentially triggered temporal summation. ${ }^{14}$ The effect of imagery on the heat test was between the baseline heat test and the CPM heat test (Figure 4), which could indicate that 1) some carryover effects of CPM impacted the imagery trials or 2) the effect of imagery is quite small.

It is possible that our study was underpowered to detect a small effect from both imagery and cognitive evaluation. Specifically, our data suggest a trend of a CPM-like effect by noxious imagery. With our current sample size, this trend (effect size 0.44 ) did not reach statistical significance. However, if we were to recruit 43 subjects, we would then be able to detect this decrease in test stimulus pain rating as a result of imagery with $80 \%$ power and an $\alpha$ of 0.05 . The effect size for that of threatening evaluation was even smaller (0.31), which would require a sample size of 67 subjects to detect it with $80 \%$ power and an $\alpha$ of 0.05 . Finally, the effect size of the reassuring evaluation was 0.018 , which is almost negligible. Therefore, it is reasonable to suspect that both the imagery and threatening evaluations might affect CPM, but the effects for both would be of much smaller magnitude compared with the effect from standard CPM.

To summarize, our results indicate that although cognitive factors (eg, evaluation) may lead to changes in the pain rating of the conditioning stimulus, they did not significantly alter the CPM magnitude in our sample. These results are consistent with the findings of multiple previous studies that report that the pain rating of the conditioning stimulus, which is subject to cognitive influences, does not predict CPM magnitude. ${ }^{12,16,25,29-31}$ Moreover, the disparate influence of cognitive evaluation on the pain rating of the conditioning stimulus itself versus CPM magnitude highlights a difference 
between static (the pain rating of the conditioning stimulus) and dynamic quantitative sensory testing (eg, CPM). The latter appears to be more robust against cognitive influences. Our findings therefore support that CPM is a reliable tool to measure endogenous analgesia.

A recent paper by Nir et $\mathrm{al}^{32}$ shows that cognitive manipulation targeted at reducing conditioning stimulus pain lessens CPM magnitude, which differs from our study. However, pronounced differences in experimental design between Nir et al's and our study (young males only vs both sexes, hot water vs cold water conditioning stimulus, and standard vs individualized conditioning stimulus) precludes a direct comparison between Nir et al's study and ours. Furthermore, the expectation manipulation performed by Nir et al was extensive, took time, and involved a placebo cream and manipulation of the conditioning stimulus. Our manipulation was much simpler and relied on verbal cues only. As such, it is conceivable that the more extreme manipulation of conditioning stimulus expectation resulted in more significant changes in CPM.

Our pilot study has two limitations. First, our sample size is limited. Although our study has enough power to replicate previous results of both the standard cold water CPM task and the evaluation task, we did not detect any effects of evaluation or imagery. We estimate that we would need to triple our sample size to have adequate power to detect the possible effects of imagery, and more than quadruple our sample size to detect the possible effect of evaluation. However, even if our results nonetheless indicate whether imagery and evaluation can affect CPM, the magnitude of their effect is much smaller than that seen using a standard cold water conditioning stimulus. This supports the robustness of the standard CPM protocol against cognitive influences. Second, although we randomized the order of evaluations and the second imagery task (and accounted for order in the analysis), order might still have influenced our results. Repeated cold water exposure from the initial thresholding test and the standard CPM task may subsequently decrease the pain ratings from the heat stimulus, because some studies show that CPM may last longer than 5 minutes. ${ }^{33,34}$ The actual effect of evaluation and imagery may thus be even smaller than what we observed. However, it is important to emphasize that order effect played a minimal role in the imagery task, given that the rating of the task performance and the magnitude of CPM-like effect from each of the imagery tasks (performed before and after real cold bath exposure) did not differ significantly.

Despite these limitations, our study adds important evidence to the growing body of research on the mechanisms of CPM. Our results strongly support the hypothesis that CPM is primarily driven by sensory input in healthy adults, and that cognitive processes such as evaluation and imagery have a relatively small effect. As such, our study confirms the robustness of the CPM protocol as a reliable means to test endogenous analgesia in healthy adults.

In future studies, it may be worthwhile to investigate the effect of CPM using the same modalities (eg, heat test and heat conditioning) versus different modalities (eg, heat test and cold conditioning). We also plan to conduct a separate examination of the effect of evaluation and imagery both to simplify our protocol and to minimize the amount of cold bath exposure experienced by our participants. The stability of sensory testing ${ }^{35}$ and utility of individualization in setting test parameters ${ }^{36}$ should also be further explored. Another important step will be to investigate whether our results remain true in patients with chronic pain, who may perform the negative evaluation and pain imagery more vividly as a result of personal experience than did our healthy young adults, who had no history of pain. The results of these future studies will inform researchers and clinicians about the ways in which psychological factors affect CPM and how these interactions contribute to the development of chronic pain.

\section{Acknowledgments}

We acknowledge funding support by NIH K24 DA029262 (SM), NIH K23 DA031808 career development grant (KJ), NIH T32 GM 89626-2 training grant (JK), and the Chris Redlich Endowment in Pain Research (SM).

\section{Disclosure}

The authors report no conflicts of interest in this work. There is no conflict of interest to be disclosed in the execution or the experiment or in the preparation of this manuscript.

\section{References}

1. Yarnitsky D. Conditioned pain modulation (the diffuse noxious inhibitory control-like effect): its relevance for acute and chronic pain states. Curr Opin Anaesthesiol. 2010;23(5):611-615

2. Le Bars D, Dickenson AH, Besson JM. Diffuse noxious inhibitory controls (DNIC). I. Effects on dorsal horn convergent neurones in the rat. Pain. 1979;6(3):283-304.

3. Le Bars D, Dickenson AH, Besson JM. Diffuse noxious inhibitory controls (DNIC). II. Lack of effect on non-convergent neurones, supraspinal involvement and theoretical implications. Pain. 1979;6(3):305-327.

4. Le Bars D, Willer J-C. Pain modulation triggered by high-intensity stimulation: implication for acupuncture analgesia? International Congress Series. 2002;1238(0):11-29.

5. Pud D, Granovsky Y, Yarnitsky D. The methodology of experimentally induced diffuse noxious inhibitory control (DNIC)-like effect in humans. Pain. 2009;144(1-2):16-19. 
6. Yarnitsky D, Arendt-Nielsen L, Bouhassira D, et al. Recommendations on terminology and practice of psychophysical DNIC testing. Eur J Pain. 2010;14(4):339.

7. Kosek E, Ordeberg G. Lack of pressure pain modulation by heterotopic noxious conditioning stimulation in patients with painful osteoarthritis before, but not following, surgical pain relief. Pain. 2000;88(1): 69-78.

8. Lautenbacher S, Rollman GB. Possible deficiencies of pain modulation in fibromyalgia. Clin J Pain. 1997;13(3):189-196.

9. Phillips K, Clauw DJ. Central pain mechanisms in chronic pain states - maybe it is all in their head. Best Pract Res Clin Rheumatol. 2011;25(2):141-154.

10. Granovsky Y. Conditioned pain modulation: a predictor for development and treatment of neuropathic pain. Curr Pain Headache Rep. 2013;17(9):361.

11. Yarnitsky D, Granot M, Nahman-Averbuch H, Khamaisi M, GranovskyY. Conditioned pain modulation predicts duloxetine efficacy in painful diabetic neuropathy. Pain. 2012;153(6):1193-1198.

12. Lautenbacher S, Roscher S, Strian F. Inhibitory effects do not depend on the subjective experience of pain during heterotopic noxious conditioning stimulation (HNCS): a contribution to the psychophysics of pain inhibition. Eur J Pain. 2002;6(5):365-374

13. Raphael KG, Janal MN, Anathan S, Cook DB, Staud R. Temporal summation of heat pain in temporomandibular disorder patients. J Orofac Pain. 2009;23(1):54-64.

14. van Wijk G, Veldhuijzen DS. Perspective on diffuse noxious inhibitory controls as a model of endogenous pain modulation in clinical pain syndromes. J Pain. 2010;11(5):408-419.

15. Edwards RR, Fillingim RB, Ness TJ. Age-related differences in endogenous pain modulation: a comparison of diffuse noxious inhibitory controls in healthy older and younger adults. Pain. 2003;101(1-2): $155-165$.

16. Granot M, Weissman-Fogel I, Crispel Y, et al. Determinants of endogenous analgesia magnitude in a diffuse noxious inhibitory control (DNIC) paradigm: do conditioning stimulus painfulness, gender and personality variables matter? Pain. 2008;136(1-2):142-149.

17. Lariviere M, Goffaux P, Marchand S, Julien N. Changes in pain perception and descending inhibitory controls start at middle age in healthy adults. Clin J Pain. 2007;23(6):506-510.

18. Washington LL, Gibson SJ, Helme RD. Age-related differences in the endogenous analgesic response to repeated cold water immersion in human volunteers. Pain. 2000;89(1):89-96.

19. Jackson T, Pope L, Nagasaka T, Fritch A, Iezzi T, Chen H. The impact of threatening information about pain on coping and pain tolerance. Br J Health Psychol. 2005;10(Pt 3):441-451.

20. Chong PS, Cros DP. Technology literature review: quantitative sensory testing. Muscle Nerve. 2004;29(5):734-747.

21. SPSS, Inc. SPSS Technical Report: Linear Mixed-Effects Modeling in SPSS: An Introduction to the MIXED Procedure. Amonk, NY: SPSS, Inc.; 2005. Available at: http://www.spss.ch/upload/1126184451_ Linear\%20Mixed\%20Effects\%20Modeling\%20in\%20SPSS.pdf. Accessed August 18, 2014.
22. Bushnell MC, Ceko M, Low LA. Cognitive and emotional control of pain and its disruption in chronic pain. Nat Rev Neurosci. 2013;14(7): 502-511.

23. deCharms RC, Maeda F, Glover GH, et al. Control over brain activation and pain learned by using real-time functional MRI. Proc Natl Acad Sci US A. 2005;102(51):18626-18631.

24. Mackey SC, Maeda F. Functional imaging and the neural systems of chronic pain. Neurosurg Clin N Am. 2004;15(3):269-288.

25. Nir RR, Granovsky Y, Yarnitsky D, Sprecher E, Granot M. A psychophysical study of endogenous analgesia: the role of the conditioning pain in the induction and magnitude of conditioned pain modulation. Eur J Pain. 2011;15(5):491-497.

26. Kakigi R. Diffuse noxious inhibitory control. Reappraisal by painrelated somatosensory evoked potentials following CO2 laser stimulation. J Neurol Sci. 1994;125(2):198-205.

27. Price DD, McHaffie JG. Effects of heterotopic conditioning stimuli on first and second pain: a psychophysical evaluation in humans. Pain. 1988;34(3):245-252.

28. Willer JC, Roby A, Le Bars D. Psychophysical and electrophysiological approaches to the pain-relieving effects of heterotopic nociceptive stimuli. Brain. 1984;107(Pt 4):1095-1112.

29. Pud D, Sprecher E, Yarnitsky D. Homotopic and heterotopic effects of endogenous analgesia in healthy volunteers. Neurosci Lett. 2005;380(3):209-213.

30. Villanueva L, Peschanski M, Calvino B, Le Bars D. Ascending pathways in the spinal cord involved in triggering of diffuse noxious inhibitory controls in the rat. J Neurophysiol. 1986;55(1):34-55.

31. Weissman-Fogel I, Sprecher E, Pud D. Effects of catastrophizing on pain perception and pain modulation. Exp Brain Res. 2008;186(1):79-85.

32. Nir RR, Yarnitsky D, Honigman L, Granot M. Cognitive manipulation targeted at decreasing the conditioning pain perception reduces the efficacy of conditioned pain modulation. Pain. 2012;153(1):170-176.

33. Tuveson B, Leffler AS, Hansson P. Time dependent differences in pain sensitivity during unilateral ischemic pain provocation in healthy volunteers. Eur J Pain. 2006;10(3):225-232.

34. Willer JC, De Broucker T, Le Bars D. Encoding of nociceptive thermal stimuli by diffuse noxious inhibitory controls in humans. J Neurophysiol. 1989;62(5):1028-1038.

35. Wilson H, Carvalho B, Granot M, Landau R. Temporal stability of conditioned pain modulation in healthy women over four menstrual cycles at the follicular and luteal phases. Pain. 2013;154(12): 2633-2638.

36. Kong JT, Johnson KA, Balise RR, Mackey S. Test-retest reliability of thermal temporal summation using an individualized protocol. J Pain. 2013;14(1):79-88. 


\section{Supplementary materials \\ Script I: Imaginary pain conditioned stimulus passage}

In the next task, we will place the thermode on your hand once again for 30 seconds. At the end of 30 seconds we will ask you to rate your pain levels. However, you will notice a bucket of ice water in the room. We would like you to imagine your right foot is situated in the ice water and that the ice water is titrated to a 5-7 pain level during the thermode testing.

If you would like to stop the test at any time for any reason, please do not hesitate to tell me during the procedure.

\section{Script 2: Reassurance orienting conditioned stimulus passage}

The cold water is a safe and effective experimental procedure.

A review of the current literature indicates that well over 1,500 experiments have used cold water with various populations including young children, pregnant women, people with chronic medical conditions, and even elderly people. The procedure is harmless, except for those persons who have indicated a medical condition listed in the consent form.

You may experience short-term discomfort or pain during and shortly after the experiment, but the procedure is safe because the water temperature has not fallen below the freezing point and because the exposure on your foot is short term. Keep this assurance in mind.

We would like you to imagine the cold water as being extremely safe. Please imagine the cold water as not being harmful.

We would like you to try to leave your foot in the cold water for as long as you can or when the proctor says you can remove your foot. However, please feel free to withdraw your foot at any point, without penalty, especially if discomfort becomes too high. (Script adapted from Jackson et al. ${ }^{1}$ )

\section{Script 3: Potential threat orienting conditioned stimulus passage}

Prolonged exposure to freezing temperatures can lead to frostbite. Frostbite is an injury to the body that is caused by freezing. Frostbite causes a loss of feeling and color in affected areas of the body. Warning signs of frostbite include sensations of tingling, throbbing, pain and numbness to the exposed limbs and flesh that has become discolored or turns blue, and unusually firm skin.

Because the frozen tissues are numb, a victim is often unaware that he/she has obtained frostbite. Nevertheless, frostbite can cause permanent damage to the body, and severe cases can lead to gangrene (when a considerable mass of body tissue dies) and the amputation of limbs.

The cold water that we will be using will not cause any of these harmful conditions. However, we would like you to imagine the cold water as being extremely harmful. Please imagine the cold water giving you frostbite and gangrene with the characteristics from above.

We would like you to leave your foot in the cold water for as long as you can or until the proctor says you can remove your foot. However, please feel free to withdraw your foot at any point, without any penalty, and especially if discomfort becomes too high. (Script adapted from Jackson et al. ${ }^{1}$ )

\section{Reference}

1. Jackson T, Pope L, Nagasaka T, Fritch A, Iezzi T, Chen H. The impact of threatening information about pain on coping and pain tolerance. Br J Health Psychol. 2005;10(Pt 3):441-451.
Journal of Pain Research

\section{Publish your work in this journal}

The Journal of Pain Research is an international, peer-reviewed, open access, online journal that welcomes laboratory and clinical findings in the fields of pain research and the prevention and management of pain. Original research, reviews, symposium reports, hypothesis formation and commentaries are all considered for publication.

\section{Dovepress}

The manuscript management system is completely online and includes a very quick and fair peer-review system, which is all easy to use. Visit http://www.dovepress.com/testimonials.php to read real quotes from published authors. 